\title{
AXIOMATIC SYSTEM DEFINING AN ORDER-EMBEDDING BETWEEN INFINITE $\sigma$-ALGEBRAS
}

\author{
NUTEFE KWAMI AGBEKO
}

Received 24 May, 2019

\begin{abstract}
The purpose of the present paper is twofold: on the one hand to set up an axiomatic system defining an order-embedding between infinite $\sigma$-algebras to generalize the powering mapping and investigate some additional necessary and sufficient condition for the postulate of powering to hold in the system, and on the other hand to provide some theoretical applications.
\end{abstract}

2010 Mathematics Subject Classification: 28A05; 28A20; 20K30; 28E05

Keywords: order-homomorphism, order-isomorphism, measurable set space, optimal measure

\section{INTRODUCTION}

It is well-known that if a $\sigma$-algebra is finite, then it is equinumerous with a power set. The question thus arises to know: Under what condition or conditions an infinite $\sigma$-algebra would be equinumerous with a power set?

By answering this question we constructed, in [2], an order-embedding (called powering mapping) from any given infinite $\sigma$-algebra into an appropriate power set and gave a necessary and sufficient condition, called the postulate of powering, which makes it onto (or a surjection).

The purpose of the present paper is twofold: on the one hand to set up an axiomatic system defining an order-embedding between infinite $\sigma$-algebras to generalize the powering mapping and investigate some additional necessary and sufficient condition for the postulate of powering to hold in the system, and on the other hand to provide theoretical applications.

But before that we shall recall some essential notions and results.

Definition $1([1,3])$. Let $(\Omega, \mathcal{F})$ be a measurable space. A set function $p: \mathcal{F} \rightarrow$ $[0,1]$ is called an optimal measure if it satisfies the following three axioms:

A1. $p$ satisfies the normalization property, i.e. $p(\Omega)=1$ and $p(\varnothing)=0$.

A2. $p$ is operation-preserving, i.e. $p(B \cup E)=p(B) \vee p(E)$ for all measurable sets $B$ and $E$. 
A3. $p$ is continuous from above, i.e. whenever $\left(E_{n}\right)_{n \in \mathbb{N}} \subset \mathcal{F}$ is a decreasing sequence, then

$$
p\left(\bigcap_{n=1}^{\infty} E_{n}\right)=\lim _{n \rightarrow \infty} p\left(E_{n}\right)=\bigwedge_{n=1}^{\infty} p\left(E_{n}\right) .
$$

The triple $(\Omega, \mathscr{F}, p)$ will be referred to as an optimal measure space.

If $p$ is an optimal measure, by a $p$-atom we mean a measurable set $H$ with $p(H)>0$ such that whenever $B \in \mathscr{F}$ and $B \subset H$, then $p(B)=p(H)$ or $p(B)=0$.

Definition 2 ([1], Definition 1.1). A $p$-atom $H$ is decomposable if there exists a subatom $B \subset H$ such that $p(B)=p(H)=p(H \backslash B)$. If no such subatom exists, the atom $H$ is then said to be indecomposable.

The Structure Theorem ([1], Theorem 1.2). Let $(\Omega, \mathcal{F}, p)$ be an optimal measure space. Then there exists a collection $\mathscr{H}(p)=\left\{H_{n}: n \in J\right\}$ of disjoint indecomposable $p$-atoms, where $J$ is some countable (i.e. finite or countably infinite) index set, such that for every measurable set $B \in \mathcal{F}$ with $p(B)>0$ we have

$$
p(B)=\max \left\{p\left(B \cap H_{n}\right): n \in J\right\} .
$$

Moreover, if $J$ is countably infinite, then the only limit point of the set $\left\{p\left(H_{n}\right): n \in J\right\}$ is 0 . (The collection of all optimal measures with countably infinite generating system is denoted by $\mathcal{P}_{\infty}$.)

We note that the Structure Theorem (see [3]) was first proved in ZFC and some two years later a similar proof was given in ZF (cf. [7]). This, in a sense, makes relevant the above question.

In the sequel we shall be dealing with infinite measurable spaces $(\Omega, \mathcal{F})$, i.e. both $\Omega$ and the $\sigma$-algebra $\mathscr{F}$ of $\Omega$ are infinite sets. (At times this will be stressed in the statements.)

Definition 3 ([2]). We say that an optimal measure $p^{*} \in \mathcal{P}_{\infty}$ is of order-one if there is a unique indecomposable $p^{*}$-atom $H$ such that $p^{*}(H)=1$. Any such atom is referred to as an order-one-atom and the set $\widetilde{\mathcal{P}_{\infty}^{1}}$ (resp. $\mathfrak{B}$ ) denotes the collection of all order-one optimal measures (resp. the collection of all order-one-atoms). We also refer to order-one as unitary.

Definition 4. The binary relation $R \subset \widetilde{\mathcal{P}_{\infty}^{1}} \times \widetilde{\mathcal{P}_{\infty}^{1}}$ is called unitary and denoted by $p_{1} R p_{2}$ if and only if there is some $p \in \widetilde{\mathcal{P}}_{\infty}^{1}$ with $H$ the corresponding unitary atom such that $p_{1}(H)=p_{2}(H)=p(H)=1$.

Remark 1 . The unitary relation is an equivalence relation, i.e. it is reflexive, symmetric and transitive. 
We note that the reflexivity and the symmetry are obvious. The transitivity is also evident, because a unitary optimal measure cannot possess two distinct unitary atoms.

If $p \in \widetilde{\mathcal{P}_{\infty}^{1}}$ with $H \in \mathfrak{B}$ its corresponding unitary atom and writing

$$
p:=\left\{q^{*} \in \widetilde{\mathcal{P}_{\infty}^{1}}: q^{*}(H)=1\right\}
$$

for the equivalence class induced by the unitary optimal measure $p$, we denote the quotient $\widetilde{\mathcal{P}_{\infty}^{1}} / R$ of $\widetilde{\mathcal{P}_{\infty}^{1}}$ modulo $R$ by $\mathcal{P}_{\infty}^{1}$, similarly as in [2].

If $A$ is a non-empty measurable set and $p \in \mathcal{P}_{\infty}^{1}$, the identity $p(A)=1$ (resp. the inequality $p(A)<1)$ will simply mean that $p^{*}(A)=1$ (resp. $\left.p^{*}(A)<1\right)$ for any representing member $p^{*} \in p$. We shall also write $p(A)=0$ to mean that $p^{*}(A)=0$ whenever $p^{*} \in p$.

Lemma 1 ([2]). Let $A, B \in \mathcal{F}$ and $p \in \mathcal{P}_{\infty}^{1}$ be arbitrary. In order that $p(A \cap B)=$ 1 it is necessary and sufficient that $p(A)=1$ and $p(B)=1$.

Remark 2 ([2]). Let $A \in \mathcal{F}$ and $p \in \mathcal{P}_{\infty}^{1}$ be arbitrary. Then the identities $p(A)=1$ and $p(\bar{A})=1$ cannot hold simultaneously, i.e. for no representing member $p^{*}$ of class $p$ the identities $p^{*}(A)=1$ and $p^{*}(\bar{A})=1$ hold at the same time.

Definition 5 ([2]). By a powering mapping we mean the following set function

$$
\begin{aligned}
\Delta: \mathcal{F} & \rightarrow \mathbb{P}\left(\mathscr{P}_{\infty}^{1}\right) \\
A & \mapsto \Delta(A):= \begin{cases}\left\{p \in \mathcal{P}_{\infty}^{1}: p(A)=1\right\} & \text { if } A \neq \varnothing \\
\varnothing & \text { if } A=\varnothing .\end{cases}
\end{aligned}
$$

Lemma 2 ([2], Lemma 2.2). Let $(\Omega, \mathcal{F})$ be an infinite measurable space. Then $\Delta(\bar{B})=\overline{\Delta(B)}$ for every set $B \in \mathcal{F}$.

It is important to note that the powering mapping is an order-embedding, and it is one-to-one.

The Postulate of Powering ([2]). If $\Gamma \in \mathbb{P}\left(\mathcal{P}_{\infty}^{1}\right) \backslash\{\varnothing\}$ and $\mathcal{C}$ denotes the collection of all unitary atoms generated by the elements of $\Gamma$, then the set $\cup \mathcal{C}$ is measurable and $\Delta(\bigcup \mathcal{C}) \subseteq \Gamma$.

Theorem 1 ([2]). The powering mapping $\Delta: \mathcal{F} \rightarrow \mathbb{P}\left(\mathcal{P}_{\infty}^{1}\right)$ is surjective if and only if the postulate of powering is valid.

\section{THE AXIOMATIC SYSTEM AND MAIN RESULTS}

Definition 6. Let $\left(\Omega_{1}, \mathcal{F}_{1}\right)$ and $\left(\Omega_{2}, \mathscr{F}_{2}\right)$ be infinite measurable spaces. A mapping $G: \mathscr{F}_{1} \rightarrow \mathscr{F}_{2}$ is said to be fundamental if it satisfies the normalization property:

$$
G\left(\Omega_{1}\right)=\Omega_{2},
$$

the difference-preserving property:

$$
G(A \backslash B)=G(A) \backslash G(B)
$$


for all $A, B \in \mathcal{F}_{1}$ and the vanishing property:

$$
G(A)=\varnothing \text { implies } A=\varnothing
$$

whenever $A \in \mathcal{F}_{1}$.

The following remark is an immediate consequence of Definition 6.

Remark 3. Let $\left(\Omega_{1}, \mathcal{F}_{1}\right)$ and $\left(\Omega_{2}, \mathcal{F}_{2}\right)$ be infinite measurable spaces and $G: \mathscr{F}_{1} \rightarrow$ $\mathcal{F}_{2}$ a fundamental mapping. Then

(1) $G(\varnothing)=\varnothing$.

(2) $G$ is a well defined mapping, i.e. if $A \in \mathscr{F}_{1}, E \in \mathcal{F}_{2}$ and $D \in \mathscr{F}_{2}$ are such that $G(A)=E$ and $G(A)=D$, then $E=D$.

(3) Identity (2.2) is equivalent to

$$
G(A \cap \bar{B})=G(A) \cap \overline{G(B)}
$$

for all $A, B \in \mathscr{F}_{1}$, where the overline denotes the complement operation.

Proof. Making the substitutions $A=B=\varnothing$ in equation (2.2), one can easily observe that $G(\varnothing)=\varnothing$. To show that $G$ is well defined, let us suppose $A \in \mathscr{F}_{1}$, $E \in \mathcal{F}_{2}$ and $D \in \mathcal{F}_{2}$ are such that $G(A)=E$ and $G(A)=D$. Then $E \backslash D=G(A) \backslash$ $G(A)=G(A \backslash A)=G(\varnothing)=\varnothing$, and similarly we can verify that $D \backslash E=\varnothing$. The conjunction of both $E \backslash D=\varnothing$ and $D \backslash E=\varnothing$ yields the desired equality $E=D$. To end the proof, we just note that (2.4) is another way of rewriting (2.2), so it is obvious, indeed.

The next basic properties about fundamental mappings are essential.

Proposition 1. Let $\left(\Omega_{1}, \mathcal{F}_{1}\right)$ and $\left(\Omega_{2}, \mathcal{F}_{2}\right)$ be infinite measurable spaces and $G$ : $\mathscr{F}_{1} \rightarrow \mathscr{F}_{2}$ be a fundamental mapping. Then for arbitrarily fixed $A, B \in \mathscr{F}_{1}$ we have:

(a) $G$ preserves the complementation, i.e. $G(\bar{B})=\overline{G(B)}$.

(b) $G$ preserves the intersection, i.e. $G(A \cap B)=G(A) \cap G(B)$.

(c) $G$ preserves the union, i.e. $G(A \cup B)=G(A) \cup G(B)$.

(d) $G$ preserves the disjointion, i.e. $G(A \cap B)=\varnothing$ if and only if $A \cap B=\varnothing$.

(e) $G(A)=G(B)$ if and only if $A=B$.

(f) $G$ is one-to-one, i.e. injective.

(g) $G$ is order-embedding, i.e. $G(A) \subseteq G(B)$ if and only if $A \subseteq B$.

Proof. Assertion (a) is immediate from both identities (2.2) and (2.1) if we replace in (2.2) $A$ with $\Omega_{1}$. To show (b) fix arbitrarily $A, B \in \mathscr{F}_{1}$. Then via identity (2.2) and assertion (a) we have

$$
\begin{aligned}
G(A \cap B) & =G(A \cap \overline{\bar{B}})=G(A \backslash \bar{B})=G(A) \backslash G(\bar{B}) \\
& =G(A) \cap \overline{\overline{G(B)}}=G(A) \cap G(B) .
\end{aligned}
$$


Assertion (c) follows from assertions (a) and (b). To show assertion (d) note that the sufficiency is trivial and the necessity follows from the vanishing property. The sufficiency condition of Assertion (e) obviously holds true. Its necessity will be shown as follows. For $A, B \in \mathscr{F}_{1}$ assume that $G(A)=G(B)$. Then by the differencepreserving property, $G(A \backslash B)=G(A) \backslash G(B)=\varnothing$, which implies via the vanishing property that $A \backslash B=\varnothing$. This means that $A \subseteq B$. Reversing the roles of $A$ and $B$ we also get $B \subseteq A$, which yields that $A=B$. Note that the fundamental mapping is one-to-one, due to assertion (e). To show the necessity of assertion (g) let $A, B \in \mathscr{F}_{1}$ and assume that $G(A) \subseteq G(B)$. Then $\varnothing=G(A) \backslash G(B)=G(A \backslash B)$, via the difference-preserving property. Applying the vanishing property we obtain the desired inclusion $A \subseteq B$. For the sufficiency proof assume that $A \subseteq B$ where $A, B \in \widetilde{F}_{1}$. Then $\varnothing=A \backslash B$. Hence by Remark 3/(1) and the difference-preserving property, $\varnothing=G(\varnothing)=G(A \backslash B)=G(A) \backslash G(B)$ which implies that $G(A) \subseteq G(B)$. To end the proof we note that Assertion (f) is a consequence of Assertions (e) or $(\mathbf{g})$.

The notion of order-embedding can be found in [4-6], for instance.

Definition 7. Let $\left(\Omega_{1}, \mathcal{F}_{1}\right)$ and $\left(\Omega_{2}, \mathcal{F}_{2}\right)$ be infinite measurable spaces. A fundamental mapping $G: \widetilde{F}_{1} \rightarrow \widetilde{F}_{2}$ is said to be tail-preserving if for every sequence $\left(A_{n}\right)_{n \in \mathbb{N}} \subset \mathscr{F}_{1}$ and any fixed number $k \in \mathbb{N}$,

$$
G\left(\bigcup_{n=k}^{\infty} A_{n}\right)=\bigcup_{n=k}^{\infty} G\left(A_{n}\right) .
$$

Remark 4. Let $\left(\Omega_{1}, \mathscr{F}_{1}\right)$ and $\left(\Omega_{2}, \mathcal{F}_{2}\right)$ be infinite measurable spaces. If the fundamental mapping $G: \mathscr{F}_{1} \rightarrow \mathcal{F}_{2}$. The the following two assertions are equivalent.

For every sequence $\left(A_{n}\right)_{n \in \mathbb{N}} \subset \mathscr{F}_{1}$ and any fixed number $k \in \mathbb{N}$,

$$
G\left(\bigcup_{n=k}^{\infty} A_{n}\right)=\bigcup_{n=k}^{\infty} G\left(A_{n}\right) \text {. }
$$

For every sequence $\left(A_{n}\right)_{n \in \mathbb{N}} \subset \mathcal{F}_{1}$,

$$
G\left(\bigcup_{n=1}^{\infty} A_{n}\right)=\bigcup_{n=1}^{\infty} G\left(A_{n}\right)
$$

Proof. Note that the conditional (a) $\Longrightarrow(\mathbf{b})$ is straightforward. To show the converse conditional suppose that for every sequence in $\mathscr{F}_{1}$ identity (2.6) is valid. Pick arbitrarily a sequence $\left(A_{n}\right)_{n \in \mathbb{N}} \subset \mathscr{F}_{1}$ and a number $k \in \mathbb{N}$. Let $B_{j}=\varnothing, j=$ $1, \ldots, k-1$ and $B_{j}=A_{j}, j \geq k$. Since $G(\varnothing)=\varnothing$, then

$$
G\left(\bigcup_{n=k}^{\infty} A_{n}\right)=G\left(\bigcup_{n=1}^{\infty} B_{n}\right)=\bigcup_{n=1}^{\infty} G\left(B_{n}\right)=\bigcup_{n=k}^{\infty} G\left(A_{n}\right) .
$$

Hence the conditional (b) $\Longrightarrow$ (a) also holds true, which completes the proof. 
Remark 5. Let $\left(\Omega_{1}, \mathcal{F}_{1}\right)$ and $\left(\Omega_{2}, \mathcal{F}_{2}\right)$ be infinite measurable spaces. If the fundamental mapping $G: \mathscr{F}_{1} \rightarrow \mathscr{F}_{2}$ is tail-preserving, then for every sequence $\left(A_{n}\right)_{n \in \mathbb{N}} \subset$ $\widetilde{F}_{1}$ and any fixed number $k \in \mathbb{N}$,

$$
G\left(\bigcap_{n=k}^{\infty} A_{n}\right)=\bigcap_{n=k}^{\infty} G\left(A_{n}\right)
$$

Proof. Fix arbitrarily a sequence $\left(A_{n}\right)_{n \in \mathbb{N}} \subset \mathscr{F}_{1}$ and a number $k \in \mathbb{N}$. Then by Definition 7 it is also true that

$$
G\left(\bigcup_{n=k}^{\infty} \overline{A_{n}}\right)=\bigcup_{n=k}^{\infty} G\left(\overline{A_{n}}\right)
$$

which yields (2.5), via De Morgan infinite identity and Proposition 1.

Proposition 2. Let $\left(\Omega_{1}, \mathcal{F}_{1}\right)$ and $\left(\Omega_{2}, \mathcal{F}_{2}\right)$ be infinite measurable spaces. If $G$ : $\widetilde{F}_{1} \rightarrow \mathscr{F}_{2}$ is a tail-preserving mapping, then each of the following assertions is valid.

i. Every sequence $\left(A_{n}\right)_{n \in \mathbb{N}} \subset \mathcal{F}_{1}$ converges increasingly if and only if $\left(G\left(A_{n}\right)\right)_{n \in \mathbb{N}} \subset \mathcal{F}_{2}$ converges increasingly.

ii. Every sequence $\left(A_{n}\right)_{n \in \mathbb{N}} \subset \mathcal{F}_{1}$ converges decreasingly if and only if $\left(G\left(A_{n}\right)\right)_{n \in \mathbb{N}} \subset \mathscr{F}_{2}$ converges decreasingly.

iii. Every sequence $\left(A_{n}\right)_{n \in \mathbb{N}} \subset \mathcal{F}_{1}$ is convergent (i.e. $\left.\liminf _{n \rightarrow \infty} A_{n}=\limsup _{n \rightarrow \infty} A_{n}\right)$ if and only if $\left(G\left(A_{n}\right)\right)_{n \in \mathbb{N}}$ is order-convergent (i.e. $\liminf _{n \rightarrow \infty} G\left(A_{n}\right)=\limsup _{n \rightarrow \infty} G\left(A_{n}\right)$ ).

Proof. The first assertion is straightforward from the assumption and Proposition $1 /(\mathrm{g})$. The second assertion can also be easily derived from the assumption, Proposition $1 /($ a) and Proposition $1 /($ g). To prove the third assertion, let us us fix an arbitrary sequence $\left(A_{n}\right)_{n \in \mathbb{N}} \subset \mathcal{F}_{1}$. Suppose that $\liminf _{n \rightarrow \infty} A_{n}=\limsup _{n \rightarrow \infty} A_{n}$. Then

$$
\bigcup_{n=1}^{\infty} \bigcap_{k=n}^{\infty} A_{k}=\bigcap_{n=1}^{\infty} \bigcup_{k=n}^{\infty} A_{k}
$$

and taking the $G$ map of these two equal sets we have by the first two parts (i. and ii.) that

$$
\bigcup_{n=1}^{\infty} \bigcap_{k=n}^{\infty} G\left(A_{k}\right)=G\left(\bigcup_{n=1}^{\infty} \bigcap_{k=n}^{\infty} A_{k}\right)=G\left(\bigcap_{n=1}^{\infty} \bigcup_{k=n}^{\infty} A_{k}\right)=\bigcap_{n=1}^{\infty} \bigcup_{k=n}^{\infty} G\left(A_{k}\right) .
$$

Consequently, $\liminf _{n \rightarrow \infty} G\left(A_{n}\right)=\limsup _{n \rightarrow \infty} G\left(A_{n}\right)$. Suppose that $\liminf _{n \rightarrow \infty} G\left(A_{n}\right)$ $=\limsup _{n \rightarrow \infty} G\left(A_{n}\right)$. Then $\bigcup_{n=1}^{\infty} \bigcap_{k=n}^{\infty} G\left(A_{k}\right)=\bigcap_{n=1}^{\infty} \bigcup_{k=n}^{\infty} G\left(A_{k}\right)$. Hence by Definition 7 and Remark 5 it follows that $G\left(\bigcup_{n=1}^{\infty} \bigcap_{k=n}^{\infty} A_{k}\right)=G\left(\bigcap_{n=1}^{\infty} \bigcup_{k=n}^{\infty} A_{k}\right)$, which implies 
$\bigcup_{\substack{n=1 \\ \text { the proof. }}}^{\infty} \bigcap_{k=n}^{\infty} A_{k}=\bigcap_{n=1}^{\infty} \bigcup_{k=n}^{\infty} A_{k}$ or equivalently, $\liminf _{n \rightarrow \infty} A_{n}=\limsup _{n \rightarrow \infty} A_{n}$. This completes

\section{FurTher CHARACTERIZATION OF THE POSTUlate OF POWERING}

Proposition 3. Let $(\Omega, \mathcal{F})$ be an infinite measurable space. Then the powering mapping $\Delta$ defined by in (1.2) is a tail-preserving fundamental mapping.

To prove Proposition 3 we will need only some basic remarks or results such as Lemma 1, Remark 2, some fundamental notions about optimal measures, as well as the following remark.

Remark 6 . Let $(\Omega, \mathcal{F})$ be an infinite measurable space and $\Delta$ the powering mapping. Then for any $A, B \in \mathcal{F}$ the following assertions are equivalent.

(1) $A \backslash B=\varnothing$.

(2) $\Delta(A \backslash B)=\varnothing$.

(3) $\Delta(A) \backslash \Delta(B)=\varnothing$.

Proof. The biconditional (1) $\Longleftrightarrow(2)$ is obvious because of Lemma 2. We show the conditional $(\mathbf{1}) \Longrightarrow(\mathbf{3})$. To this end assume by the contrapositive that $\Delta(A) \backslash$ $\Delta(B) \neq \varnothing$ and pick an arbitrary class $p \in \Delta(A) \backslash \Delta(B)$. Then $p \in \Delta(A)$ and $p \notin \Delta(B)$. Thus necessarily $p \in \overline{\Delta(B)}=\Delta(\bar{B})$, via Lemma 2. But since also $p \in$ $\Delta(A)$ we have that $p(A)=p(\bar{B})=1$ or equivalently $p(A \cap \bar{B})=1$ due to Lemma 1. Consequently, $p \in \Delta(A \cap \bar{B})=\Delta(A \backslash B)$, implying that $\Delta(A \backslash B) \neq \varnothing$. One can thus easily deduce from the definition of $\Delta$ that $A \backslash B \neq \varnothing$. Finally, we show the conditional (3) $\Longrightarrow(2)$. To this end assume by the contrapositive that $\Delta(A \backslash B) \neq \varnothing$ and pick arbitrarily a class $p \in \Delta(A \backslash B)$. Then $p(A)=1$ and $p(\bar{B})=1$. But $p(\bar{B})=1$ implies as above that $p \in \Delta(\bar{B})=\overline{\Delta(B)}$. Consequently, $p \in \Delta(A) \cap$ $\overline{\Delta(B)}=\Delta(A) \backslash \Delta(B)$ which proves that $\Delta(A) \backslash \Delta(B) \neq \varnothing$. This completes the proof.

Proof of Proposition 3. First we show that $\Delta$ is a fundamental mapping. In fact, pick arbitrarily a class $p \in \mathcal{P}_{\infty}^{1}$. Then due to the fact that every optimal measure assigns to $\Omega$ the value 1 it follows that $p(\Omega)=1$, i.e. $p \in \Delta(\Omega)$, which means that $\mathcal{P}_{\infty}^{1} \subseteq \Delta(\Omega)$. As the reverse inclusion is straightforward we conclude that the powering mapping satisfies the normalization property. Next, we show that $\Delta$ is a difference-preserving mapping, i.e. $\Delta(A \backslash B)=\Delta(A) \backslash \Delta(B)$ for all $A, B \in \mathcal{F}$. We have two cases to consider.

(a) In the first case, suppose $A \backslash B=\varnothing$. Then due to Remark 6 identity $\Delta(A \backslash B)=$ $\Delta(A) \backslash \Delta(B)$ follows.

(b) In the second case, suppose $A \backslash B \neq \varnothing$, and fix arbitrarily $p \in \Delta(A \backslash B)$. Then $p(A \cap \bar{B})=p(A \backslash B)=1$, and so Lemma 1 yields that both identities $p(A)=1$ 
and $p(\bar{B})=1$ are sastisfied. Thus $p \in \Delta(A)$ and $p \in \Delta(\bar{B})=\overline{\Delta(B)}$. Then $p \in \Delta(A) \cap \overline{\Delta(B)}$. Consequently, $\Delta(A \backslash B) \subseteq \Delta(A) \cap \overline{\Delta(B)}=\Delta(A) \backslash \Delta(B)$. To show the reverse inclusion fix arbitrarily a class $p \in \Delta(A) \backslash \Delta(B)$. Then $p \in$ $\Delta(A)$ and $p \notin \Delta(B)$. Thus $p \in \Delta(A \cap \bar{B})$ and so $\Delta(A) \backslash \Delta(B) \subseteq \Delta(A \cap \bar{B})=$ $\Delta(A \backslash B)$. Consequently, $\Delta(A \backslash B)=\Delta(A) \backslash \Delta(B)$.

Therefore, $\Delta$ satisfies the difference-preserving property. The vanishing property is obvious from Remark 6 . We have thus shown that $\Delta$ is a fundamental mapping.

Finally, we prove that $\Delta$ is tail-preserving. To this end select arbitrarily a sequence $\left(A_{n}\right)_{n \in \mathbb{N}} \subset \mathcal{F}$ and a number $k \in \mathbb{N}$. We have two cases to go into:

(i) In the first case, suppose $\bigcup_{n=k}^{\infty} A_{n}=\varnothing$, which is equivalent to saying that $A_{n}=\varnothing$ for all $n \in \mathbb{N}, n \geq k$. Hence by definition $\Delta\left(\bigcup_{n=k}^{\infty} A_{n}\right)=\varnothing$. Because of the above two equivalent assertions, for all $n \in \mathbb{N}, n \geq k$ we have $A_{n}=\varnothing$ which yields via the definition of $\Delta$ that $\Delta\left(A_{n}\right)=\varnothing$ whenever $n \geq k$. Consequently,

$$
\Delta\left(\bigcup_{n=k}^{\infty} A_{n}\right)=\varnothing=\bigcup_{n=k}^{\infty} \Delta\left(A_{n}\right)
$$

(ii) In the second case, suppose $\bigcup_{n=k}^{\infty} A_{n} \neq \varnothing$. Then by definition

$$
\Delta\left(\bigcup_{n=k}^{\infty} A_{n}\right)=\left\{p \in \mathcal{P}_{\infty}: p\left(\bigcup_{n=k}^{\infty} A_{n}\right)=1\right\} \neq \varnothing
$$

Fix arbitrarily a class $p \in \Delta\left(\bigcup_{n=k}^{\infty} A_{n}\right)$ and a unitary optimal measure $p^{*} \in p$. Then $p^{*}\left(\bigcup_{n=k}^{\infty} A_{n}\right)=1$. Clearly, the increasing sequence $\left(p^{*}\left(\bigcup_{n=k}^{j} A_{n}\right)\right)_{j \geq k}$ tends discretely to $p^{*}\left(\bigcup_{n=k}^{\infty} A_{n}\right)=1$, i.e. a natural number $m \geq k$ can be found such that $p^{*}\left(\bigcup_{n=k}^{m} A_{n}\right)=1$, (see the proof of [3, Lemma 2.1]). Hence there is an index $j_{0} \in\{k, k+1, \ldots, m\}$ such that $p^{*}\left(A_{j_{0}}\right)=1$ and thus $p\left(A_{j_{0}}\right)=1$, 


$$
\begin{aligned}
\text { i.e. } p \in \Delta\left(A_{j_{0}}\right) \subset \bigcup_{n=k}^{\infty} \Delta\left(A_{n}\right) \text {. Consequently, } \\
\qquad \Delta\left(\bigcup_{n=k}^{\infty} A_{n}\right) \subset \bigcup_{n=k}^{\infty} \Delta\left(A_{n}\right) .
\end{aligned}
$$

The reverse inclusion can be easily derived from Proposition $1 / \mathrm{g}$, because $\Delta$ has been proved to be a fundamental mapping.

Therefore, we conclude on the validity of the argument.

Theorem 2. Let $(\Omega, \mathcal{F})$ be an infinite measurable space and $\Delta: \mathcal{F} \rightarrow \mathbb{P}\left(\mathcal{P}_{\infty}^{1}\right)$ be a powering mapping. Then the following assertions are equivalent.

C1. The postulate of powering is a valid statement.

C2. The powering mapping $\Delta$ is onto.

C3. There is an infinite set $X$ such that the power set $\mathbb{P}(X)$ and the $\sigma$-algebra $\mathcal{F}$ are tail-preserving order-isomorphic, i.e. there is a tail-preserving fundamental mapping $\beta: \mathbb{P}(X) \rightarrow \mathcal{F}$ which is also a bijection.

Proof. The proof of the biconditional $\mathbf{C 1} \Longleftrightarrow \mathbf{C 2}$ can be found in [2].

The conditional $\mathbf{C} 2 \Longrightarrow \mathbf{C} 3$ is trivial. In fact, since the powering mapping is one-toone, Assertion $\mathbf{C} 2$ obviously implies that the powering mapping $\Delta: \mathscr{F} \rightarrow \mathbb{P}\left(\mathcal{P}_{\infty}^{1}\right)$ is a bijection, and due to Proposition 3 it is also a tail-preserving fundamental mapping. Hence $\mathbf{C} 3$ holds true if we choose $X$ to be $\mathcal{P}_{\infty}^{1}$. It remains to check the validity of the implication $\mathbf{C 3} \Longrightarrow \mathbf{C 1}$. In fact, for this purpose assume the existence of an infinite set $X$ and some tail-preserving fundamental mapping $\beta: \mathbb{P}(X) \rightarrow \mathcal{F}$. We aim to prove the validity of the postulate. Pick arbitrarily a $\Gamma \in \mathbb{P}\left(\mathcal{P}_{\infty}^{1}\right) \backslash\{\varnothing\}$ with $\mathcal{C}$ its corresponding collection of unitary atoms. Let

$$
X_{\mathcal{C}}:=\{x \in X: \beta(\{x\}) \in \mathcal{C}\} .
$$

Clearly, $X_{\complement} \in \mathbb{P}(X)$. Hence $\beta\left(X_{\complement}\right) \in \mathcal{F}$. Next, we show that $\beta\left(X_{\complement}\right)=\bigcup \mathscr{C}$. In fact, since $\beta$ is a bijection, for every $H \in \mathcal{C}$ there is a unique $x \in X_{\mathcal{C}}$ such that $\beta^{-1}(H)=$ $\{x\} \subset X_{\ell}$. As $\beta$ preserves the order, the inclusion $H \subset \beta\left(X_{\complement}\right)$ follows, implying that Ue $\subset \beta\left(X_{\ell}\right)$. Conversely, for arbitrary $x \in X_{\mathcal{C}}$ there is a unique $H \in \mathcal{C}$ such that $\beta(\{x\})=H \subset \bigcup \mathcal{C}$. Hence $\{x\} \subset \beta^{-1}(\bigcup \mathcal{C})$, which implies that $X_{\mathcal{C}} \subseteq \beta^{-1}(\bigcup \mathcal{C})$ or, equivalently $\beta\left(X_{\ell}\right) \subseteq \cup \varphi$. We have thus proved that $\beta\left(X_{\ell}\right)=\bigcup \mathscr{C}$. This means that $\bigcup \mathscr{C} \in \mathcal{F}$. To complete the proof pick arbitrarily some $p \in \Delta(\bigcup \mathscr{C})$. Then $p(\bigcup \mathcal{C})=1$, yielding $p(H)=1$ for some $H \in \mathcal{C}$. Then $p \in \Gamma$, which implies that $\Delta(\bigcup \mathcal{C}) \subseteq \Gamma$. Therefore, the postulate holds true, indeed. This was to be proven.

Assume that there is an infinite set $X$ such that its power set $\mathbb{P}(X)$ and the $\sigma$ algebra $\mathcal{F}$ are tail-preserving order-isomorphic, with $\beta: \mathbb{P}(X) \rightarrow \mathscr{F}$ the acting bijective tail-preserving fundamental mapping. The question arises to know the totallity of functions $p: \mathcal{F} \rightarrow[0,1]$ such that $p \circ \beta: \mathbb{P}(X) \rightarrow[0,1]$ is an optimal measure on the measurable space $(X, \mathbb{P}(X))$. 
Proposition 4. Let $(\Omega, \mathcal{F})$ be an infinite measurable space. Assume there is an infinite set $X$ such that its power set $\mathbb{P}(X)$ and the $\sigma$-algebra $\mathcal{F}$ are tail-preserving isomorphic, with $\beta: \mathbb{P}(X) \rightarrow \mathscr{F}$ the acting order-isomorphism. The following assertions hold true.

(i) If $p$ is an optimal measure and $H \in \mathcal{F}$ an indecomposable p-atom, then $\beta^{-1}(H) \in \mathbb{P}(X)$ is an indecomposable $p \circ \beta$-atom.

(ii) If $p \circ \beta$ is an optimal measure and $H^{(X)} \in \mathbb{P}(X)$ an indecomposable $p \circ \beta$ atom, then $\beta\left(H^{(X)}\right) \in \mathscr{F}$ is an indecomposable p-atom.

Proof. Suppose that $p$ is an optimal measure on $\mathscr{F}$ and $H \in \mathscr{F}$ is an indecomposable $p$-atom. Then there uniquely exists an $H^{(X)} \in \mathbb{P}(X)$ such that $\beta\left(H^{(X)}\right)=H$. We show that $H^{(X)}$ is a $p \circ \beta$-atom. In fact, because $\beta$ is order preserving, for any $B^{(X)} \subset H^{(X)}$ it is true that $\beta\left(B^{(X)}\right) \subset H$, so that either $p\left(\beta\left(B^{(X)}\right)\right)=0$ or $p\left(\beta\left(B^{(X)}\right)\right)=p(H)$. Consequently, either $p \circ \beta\left(B^{(X)}\right)=0$ or $p \circ \beta\left(B^{(X)}\right)=$ $p \circ \beta\left(H^{(X)}\right)$, i.e. $H^{(X)}$ is a $p \circ \beta$-atom. Moreover, suppose $H$ is an indecomposable $p$-atom, but there is some $\beta\left(B^{(X)}\right) \subset H$ with $p \circ \beta\left(B^{(X)}\right)=p \circ \beta\left(H^{(X)}\right)=p \circ$ $\beta\left(H^{(X)} \backslash B^{(X)}\right), \quad$ or $\quad$ equivalently, $\quad p\left(\beta\left(B^{(X)}\right)\right)=p\left(\beta\left(H^{(X)}\right)\right)=$ $p\left(\beta\left(H^{(X)}\right) \backslash \beta\left(B^{(X)}\right)\right)$. This, however, violates that $H$ is an indecomposable $p$-atom, since $\beta\left(B^{(X)}\right)$ and $\beta\left(H^{(X)}\right) \backslash \beta\left(B^{(X)}\right)$ are subsets of $H$. We shall conclude the proof by pointing out that the second part can be similarly proved.

\section{AN EXAMPLE OF BIJECTIVE TAIL-PRESERVING FUNDAMENTAL MAPPING WITH APPLICATION}

Theorem 3. Let $\Omega$ be an infinite set and $\mathscr{H}:=\left\{H_{n}: n \in \mathbb{N}\right\}$ a countably infinite partition of $\Omega$. Denote by $\sigma(\mathcal{H})$ the $\sigma$-algebra generated by $\mathcal{H}$. Then the correspondence $\varphi: \sigma(\mathscr{H}) \rightarrow \mathbb{P}(\mathbb{N})$, defined by

$$
\varphi(A)=\left\{n \in: \mathbb{N}: H_{n} \subset A\right\}, A \in \sigma(\mathcal{H}),
$$

is a bijective tail-preserving fundamental mapping.

Before we prove the theorem we first make the following obvious remark.

Remark 7. Let $\Omega$ be an infinite set and $\mathscr{H}:=\left\{H_{n}: n \in \mathbb{N}\right\}$ a countably infinite partition of $\Omega$. Denote by $\sigma(\mathscr{H})$ the $\sigma$-algebra generated by $\mathscr{H}$. Then the following assertions are valid.

(i)

$$
\sigma(\mathscr{H})=\left\{\bigcup_{n \in \boldsymbol{K}} H_{n}: \boldsymbol{K} \subset \mathbb{N}\right\}
$$


(ii) For all sets $A \in \sigma(\mathscr{H})$ and $H \in \mathscr{H}$, either $H \cap A=H$ or $H \cap A=\varnothing$.

(iii) Let sequence $\left(A_{n}\right)_{n \in \mathbb{N}} \subset \sigma(\mathscr{H})$ and number $k \in \mathbb{N}$ be arbitrary. Then for any given $H \in \mathscr{H}$ for which $H \subset \bigcup_{m \geq k} A_{m}$, there exists an index $n \geq k$ such that $H \subset A_{n}$.

Proof Theorem 3. The proof is split into three steps. We would like to inform the reader that Remark 7 will be used several times in the proof without always mentioning it.

Step 1. We show that $\varphi$ is a bijection. In fact, let $A, B \in \sigma(\mathscr{H})$ and assume that $\varphi(A)=\varphi(B)$. Then $A=\bigcup_{n \in \varphi(A)} H_{n}=\bigcup_{n \in \varphi(B)} H_{n}=B$, i.e. $\varphi$ is one-to-one. Next, let $\boldsymbol{K} \in \mathbb{P}(\mathbb{N})$ be arbitrary and denote $A:=\bigcup_{n \in \boldsymbol{K}} H_{n}$. Clearly, $A \in \sigma(\mathcal{H})$, meaning that $\varphi(A)=\boldsymbol{K}$, i.e. $\varphi$ is onto. Therefore, $\varphi$ is a bijection.

Step 2. In this step, we prove that $\varphi$ is a fundamental mapping. We need to verify the normalization, the difference-preserving and the vanishing properties, as follows.

(a) By definition it is obvious that $\varphi(\Omega)=\mathbb{N}$, i.e. the normalization property holds.

(b) We show that $\varphi(A \backslash B)=\varphi(A) \backslash \varphi(B)$, for all $A, B \in \sigma(\mathcal{H})$. In fact, fix arbitrarily an $n \in \varphi(A \backslash B)$. Then $H_{n} \subset A \backslash B=A \cap \bar{B}$, i.e. $H_{n} \subset A$ and $H_{n} \subset \bar{B}$, or equivalently, $n \in \varphi(A)$ and $n \notin \varphi(B)$, or equivalently $n \in \varphi(A) \backslash \varphi(B)$ for all $n \in \varphi(A \backslash B)$. Hence $\varphi(A \backslash B) \subset \varphi(A) \backslash \varphi(B)$. To show that $\varphi(A) \backslash \varphi(B) \subset$ $\varphi(A \backslash B)$, pick arbitrarily an $n \in \varphi(A) \backslash \varphi(B)$. Then necessarily $H_{n} \subset A$ and $H_{n} \cap B=\varnothing$, by Remark 7. Hence $H_{n} \subset A$ and $H_{n} \subset \bar{B}$, or equivalently, $H_{n} \subset A \cap \bar{B}$, meaning that $n \in \varphi(A \cap \bar{B})$, and hence $\varphi(A) \backslash \varphi(B) \subset \varphi(A \backslash B)$. Therefore, $\varphi(A \backslash B)=\varphi(A) \backslash \varphi(B)$, i.e. $\varphi$ satisfies the difference-preserving property.

(c) Note that $\varphi$ obviously satisfies the vanishing property.

Step 3. We are left to check that $\varphi$ satisfies the tail-preserving property. To do this pick arbitrarily a sequence $\left(A_{n}\right)_{n \in \mathbb{N}} \subset \sigma(\mathscr{H})$ and a number $k \in \mathbb{N}$. Also fix any number

$$
t \in \varphi\left(\bigcup_{m=k}^{\infty} A_{m}\right)=\left\{u \in \mathbb{N}: H_{u} \subset \bigcup_{m=k}^{\infty} A_{m}\right\} .
$$

Then $H_{t} \subset \bigcup_{m=k}^{\infty} A_{m}$ implying the existence of an index $m_{0} \geq k$ for which $H_{t} \subset A_{m_{0}}$. So that

$$
t \in \varphi\left(A_{m_{0}}\right) \subset \bigcup_{m=k}^{\infty} \varphi\left(A_{m}\right)
$$


We have thus proved that

$$
\varphi\left(\bigcup_{m=k}^{\infty} A_{m}\right) \subset \bigcup_{m=k}^{\infty} \varphi\left(A_{m}\right) .
$$

To show the converse inclusion, let $t \in \bigcup_{m=k}^{\infty} \varphi\left(A_{m}\right)$ be arbitrarily fixed. Then there is an index $m_{0} \geq k$ such that $t \in \varphi\left(A_{m_{0}}\right)$. Note that $\varphi$, being a fundamental mapping, it is also an order-embedding. Thus, $\varphi\left(A_{m_{0}}\right) \subset \varphi\left(\bigcup_{m=k}^{\infty} A_{m}\right)$ and, as $t \in \varphi\left(A_{m_{0}}\right)$ it follows that $t \in \varphi\left(\bigcup_{m=k}^{\infty} A_{m}\right)$. Hence $\bigcup_{m=k}^{\infty} \varphi\left(A_{m}\right) \subset \varphi\left(\bigcup_{m=k}^{\infty} A_{m}\right)$. Consequently,

$$
\varphi\left(\bigcup_{m=k}^{\infty} A_{m}\right)=\bigcup_{m=k}^{\infty} \varphi\left(A_{m}\right)
$$

which is to say that $\varphi$ is tail-preserving. This completes the proof.

Corollary 1. Let $\mathcal{F}_{0} \subset \mathbb{P}(\mathbb{N})$ be an infinite sub- $\sigma$-algebra. Then $\mathcal{F}_{0}$ is equinumerous with the power set $\mathbb{P}(\mathbb{N})$.

Proof. Let $\mathscr{H}:=\left\{H_{n}: n \in \mathbb{N}\right\}$ be a countably infinite $\mathscr{F}_{0}$-measurable partition of $\mathbb{N}$. Then by Theorem $3, \sigma(\mathscr{H})$ is equinumerous with the power set $\mathbb{P}(\mathbb{N})$. But since $\sigma(\mathscr{H})$ is obviously an infinite sub- $\sigma$-algebra of $\mathscr{F}_{0}$, it must follow that $\mathscr{F}_{0}$ itself is equinumerous with the power set $\mathbb{P}(\mathbb{N})$. This was to be proven.

We can thus remark that every sub- $\sigma$-algebra contained in the power set $\mathbb{P}(\mathbb{N})$ is equinumerous either with the power set itself or with a finite power set.

Corollary 2. The powering mapping defined on the $\sigma$-algebra generated by a countably infinite partition of an infinite set $\Omega$ satisfies the postulate of powering and thus is onto.

We shall end our paper with the following application involving measure theory.

Proposition 5. Let $\left(\Omega_{1}, \mathcal{F}_{1}\right)$ and $\left(\Omega_{2}, \mathcal{F}_{2}\right)$ be infinite measurable spaces. Assume $G: \mathscr{F}_{1} \rightarrow \mathscr{F}_{2}$ is a bijective tail-preserving fundamental mapping. Then the following two assertions are valid. A function $\mu: \widetilde{F}_{2} \rightarrow[0,1]$ is a probability measure if and only if the composition mapping $\mu \circ G: \widetilde{F}_{1} \rightarrow[0,1]$ is also a probability measure.

Proof. Assume that $\mu: \mathscr{F}_{2} \rightarrow[0,1]$ is a probability measure, i.e.

$$
\mu\left(\Omega_{2}\right)=1, \text { and }
$$


(2)

$$
\mu\left(\bigcup_{n=1}^{\infty} B_{n}\right)=\sum_{n=1}^{\infty} \mu\left(B_{n}\right)
$$

for every sequence of pairwise disjoint measurable sets $\left(B_{n}\right)_{n \in \mathbb{N}}$. Then $1=\mu\left(\Omega_{2}\right)=$ $\mu\left(G\left(\Omega_{1}\right)\right)$, i.e. $(\mu \circ G)\left(\Omega_{1}\right)=1$. Next, let $\left(A_{n}\right)_{n \in \mathbb{N}} \subset \mathscr{F}_{1}$ be any sequence of pairwise disjoint measurable sets. Then there uniquely exists a sequence $\left(B_{n}\right)_{n \in \mathbb{N}} \subset$ $\widetilde{F}_{2}$ such that $B_{n}=G\left(A_{n}\right)$ for all $n \in \mathbb{N}$ (because $G$ is a bijection). The $B_{n}$ 's so obtained are pairwise disjoint because $G$ preserves the disjointion. Consequently,

$$
\begin{aligned}
\sum_{n=1}^{\infty}(\mu \circ G)\left(A_{n}\right) & =\sum_{n=1}^{\infty} \mu\left(B_{n}\right)=\mu\left(\bigcup_{n=1}^{\infty} B_{n}\right)=\mu\left(\bigcup_{n=1}^{\infty} G\left(A_{n}\right)\right) \\
& =\mu\left(G\left(\bigcup_{n=1}^{\infty} A_{n}\right)\right)=(\mu \circ G)\left(\bigcup_{n=1}^{\infty} A_{n}\right) .
\end{aligned}
$$

We have thus proved that $\mu \circ G$ is a probability measure on $\mathscr{F}_{1}$. The proof of the converse conditional can be similarly carried out, so it is left.

\section{ACKNOWLEDGEMENT}

I would like to express my sincere gratitude to Prof. Zoltán Boros for his suggestions.

\section{REFERENCES}

[1] N. K. Agbeko, "On the structure of optimal measures and some of its applications," Publ. Math. Debrecen, vol. 46, no. 1-2, pp. 79-87, 1995.

[2] N. K. Agbeko, "Mapping bijectively $\sigma$-algebras onto power sets," Math. Notes (Miskolc), vol. 2, no. 2, pp. 85-92, 2001, [http://mat76.mat.uni-miskolc.hu/mnotes/contents/2/2/].

[3] N. Agbeko, "On optimal averages," Acta Math. Hungar., vol. 63, no. 2, pp. 133-147, 1994.

[4] G. Birkhoff, Lattice theory, 3rd ed., ser. American Mathematical Society Colloquium Publications. American Mathematical Society, Providence, R.I., 1979, vol. 25.

[5] N. Bourbaki, Elements of mathematics. Theory of sets, ser. Translated from the French. Hermann, Publishers in Arts and Science, Paris; Addison-Wesley Publishing Co., Reading, Mass.-LondonDon Mills, Ont., 1968.

[6] B. A. Davey, Introduction to lattices and order, ser. Cambridge Mathematical Textbooks. Cambridge University Press, Cambridge, 1990.

[7] I. Fazekas, "A note on optimal measures," Publ. Math. Debrecen, vol. 51, no. 3-4, pp. 273-277., 1997.

Author's address

Nutefe Kwami Agbeko

Institute of Mathematics, University of Miskolc, 3515 Miskolc-Egyetemváros, Hungary

E-mail address: matagbek@uni-miskolc.hu 